\title{
Preoperative COVID-19 Testing for Elective Ophthalmological Procedure in a Tertiary Health Care Centre: Our Experience During the Pandemic
}

\author{
Mrityunjay Singh \\ Harinder Singh Sethi \\ Sukriti Gupta \\ Ram Kishan Duvesh \\ Mayuresh Naik (D)
}

Department of Ophthalmology, VMMC and Safdarjung Hospital, New Delhi, India
Correspondence: Ram Kishan Duvesh Department of Ophthalmology, VMMC and Safdarjung Hospital, New Delhi, India, Tel +9l 9654530391

Email mayureshpnaik@gmail.com
Background: To study the prevalence of coronavirus infection among asymptomatic patients requiring eye surgery and the role of screening in prevention of spread of infection among the healthcare workers.

Design: The prospective observational study was conducted in the Ophthalmology department of a tertiary care center in Delhi from September 2020 to December 2020.

Setting: Patients requiring elective ophthalmological procedures in a tertiary care hospital were screened for coronavirus using the RT-PCR method. Testing methods and results were documented.

Results: Among the 218 asymptomatic patients posted for elective surgery in that period, 16 (7.3\%) were found to be positive for COVID-19. Those who tested positive were advised home isolation and surgery was postponed for the next 14 days. No complications were reported in these patients. One health-care worker also tested positive for COVID-19 during our study period.

Conclusion: In our study, 1 out of 14 asymptomatic patients were found to be carriers for the novel virus. Asymptomatic COVID-infected patients may lead to transmission of the virus inside the hospital among the visiting patients and hospital staff while they have no adverse effect on the surgery and its outcome.

Keywords: COVID-19, elective procedure, phacoemulsification

\section{Introduction}

The COVID-19 pandemic and post-surge planning highlights the significance of preoperative optimization in a way that was previously unrecognized in the perioperative period. Since the pandemic measures were taken to protect healthcare staff from infection, millions of surgeries have been postponed owing to this pandemic. Globally, approximately 28.4 million elective surgeries have been postponed due to the pandemic. ${ }^{1}$ Robust COVID testing has made it possible to diagnose asymptomatic patients. After 9 months of the pandemic, the government of India has been trying to restart elective surgeries along with the safety of healthcare personnel and the patients. A major concern to this is that COVID-19 patients can visit the hospital in the asymptomatic transmission phase and identifying such asymptomatic patients is challenging. Therefore, COVID-19 testing before elective surgeries has been indicated by some international authorities. ${ }^{2}$ The Indian Council of Medical Research guidelines in June 2020 stated that COVID-19 screening for asymptomatic patients coming for elective surgeries should be performed for 
otorhinolaryngology surgery, neurosurgery, dental procedures and for non-surgical interventions such as upper gastrointestinal endoscopy, bronchoscopy and for many other diagnostic procedures. ${ }^{3}$

The All India Ophthalmology Society had laid down guidelines for ophthalmologists to be followed during the pandemic in April 2020. ${ }^{4}$ The American Academy of Ophthalmology recommended on March 18, 2020 that all routine ophthalmological procedures should be stopped and only urgent procedures should be done. However it was left to each doctor's judgement to define urgent procedures. ${ }^{5}$ Meanwhile the British Oculoplastic Surgery Society (BOSS) highlighted the formation of bioaerosols in ocular surgeries. Those surgeries involving the nasal, lacrimal or sinus cavity were at higher risk. ${ }^{6}$

Kannan et al. studied prevalence of COVID-19 infection among patients undergoing vitreoretinal surgery. They concluded that among patients visiting high volume ophthalmic centres in the near future, approximately 1 in 45 patients may be asymptomatic, SARS-CoV-2 reverse transcriptionpolymerase chain reaction (RT-PCR) positive. ${ }^{7}$ A drawback in this study was that the testing was not conducted preoperatively in ophthalmic procedures which are of lesser duration related to cataract, cornea and glaucoma. Hence this study was taken to highlight the importance of COVID-19 testing as a routine before any operative procedure in order to prevent transmission of the disease and for the protection of healthcare workers in this pandemic.

\section{Materials and Methods}

The retrospective observational study was conducted in the Department of Ophthalmology of a tertiary care center in New Delhi from September 2020 to March 2020. Ethical clearance was obtained from Ethical Clearance committee, Institutional review board, VMMC \& Safdarjung Hospital (IEC.IRB/VMMC/SJH/09/2020-121). Informed consent was taken from all the patients for COVID-19 testing, participation in the study and intervention separately. The study was conducted in accordance with the Declaration of Helsinki.

At the doorstep of the outpatient department, personnel were deputed who questioned every patient regarding symptoms like fever, cough, breathlessness and contact with any COVID-positive patient recently. Temperature was checked using infrared thermometer and status of patient was checked using the Aarogya Setu app.

Patients showing any suspected symptoms for COVID19 infection were referred to a fever clinic in the hospital. Patients were asked to follow COVID-19 safety protocols i.e. wearing a mask, social distancing, using hand sanitizers. On the other hand, doctors protected themselves by wearing N95 masks, protective disposable gowns, gloves, head cap and face shields. Slit lamp examinations were done using a detachable shield attached to the viewing arm of a slit lamp. Patients were examined in the general outpatient Department of Ophthalmology and were then sent to respective special clinics according to their signs and symptoms.

Patients posted for major surgeries underwent a mandatory COVID-19 RT-PCR testing. Ophthalmology residents were trained for taking nasal and oral samples for RT-PCR and a member of the nursing staff was deputed to carry the samples to microbiology lab under aseptic conditions. Samples were taken one day prior to surgery free of cost and results were obtained using reverse transcriptionpolymerase chain reaction (RT-PCR) method. Patients posted for surgery under general anesthesia were advised to have chest X-ray and pre-anesthetic clearance was taken. Proper protective equipment and all necessary precautions as laid out by the All India Ophthalmology Society were taken for testing the patients. The sample were then sent in a viral transport medium to the microbiology lab of Safdarjung Hospital. The test was performed on RT PCR kits (Covidsure) using SARSCoV-2 Co Primer sets designed to detect RNA (RdRp gene) from SARS-CoV-2 in the upper respiratory tract.

The patients undergoing testing were advised home isolation until the surgery. Only those patients who were negative by RT-PCR method were taken up for surgery the next day. Elective surgeries were resumed in the hospital from September 2020 with proper precautions as per ICMR guidelines. $^{3}$

In cases where a patient tested positive, he/she was counselled regarding the nature of the disease and were advised for home isolation with self-monitoring as they were asymptomatic. They were asked to review after 14 days for repeat COVID-19 RT-PCR test. The surgeries which were postponed, were conducted later after testing negative for the novel virus.

The healthcare workers were also repeatedly checked for fever cough and breathlessness. One healthcare worker became infected with the novel virus and was home quarantined for 14 days.

\section{Results}

Between September 2020 to December 2020, a total of 218 patients required elective surgery at Safdarjung Hospital related to their eyes. The mean age of the patients was 61 \pm 10.5 year ( 2 months -75 years). Amongst those, 97 were males and 121 females (Table 1). Testing was done in all the 
Table I Demographic Data

\begin{tabular}{|l|l|}
\hline $\begin{array}{l}\text { Average Age of Study } \\
\text { Population }\end{array}$ & 6I Years (2 Months - 75 Years) \\
\hline Male: Female & $4: 5$ \\
Number of positive patients & $16(7.3 \%)$ \\
Number of surgeries done & $192(88.07 \%)$ \\
Number of cases postponed & $26(11.93 \%)$ \\
\hline
\end{tabular}

218 patients $(100 \%)$. Of these patients, $16(7.3 \%)$ were found to be positive for COVID-19. All these 16 patients were asymptomatic and their surgeries were postponed. The median period reported for an asymptomatic patient to become negative for viral nucleic acid is 9.5 days and the longest is up to 21 days. $^{8}$ All the patients who tested positive for COVID-19 were informed, and were home quarantined for 2 weeks (Table 2). Contact tracing was also done and their contacts were tested for COVID-19 infection.

Out of 202 COVID-19 negative patients, 192 (95.0\%) surgeries were conducted (Table 3) and $10(5 \%)$ were postponed in this period of 3 months. Out of these 10 patients, 5 of them were postponed due to uncontrolled diabetes, 3 were postponed due to hypertension and the remaining 2 due to conjunctivitis. 186 (97\%) cases were done with local anesthesia and remaining $6(3 \%)$ cases were done under general anesthesia.

\section{Discussion}

During the COVID-19 pandemic, screening, testing and contact tracing have been the foremost strategies to control
Table 3 Types of Surgeries Performed During the Study Period

\begin{tabular}{|l|l|}
\hline Surgery & Number of Patients \\
\hline Phacoemulsification & 150 \\
Small incision cataract surgery & 10 \\
Pterygium excision & 11 \\
Lid mass excision & 4 \\
Corneal foreign body removal & 4 \\
Trabeculectomy & 10 \\
Squint surgery & 3 \\
\hline
\end{tabular}

the spread of the virus. With extensive testing and contact tracing, India is still witnessing a rise in cases. Asymptomatic COVID cases are the biggest hurdle in preventing this pandemic.

This study was conducted to evaluate the current evidence with opinion from clinical experts to draft guidelines for active COVID-19 screening in a hospital setting. It is seen that COVID-19 patients undergoing surgical procedures have a higher risk of mortality than the general population. ${ }^{9}$ Hence surgeries are being avoided in COVID-positive patients which necessitates testing in all cases.

Testing all patients before taking up for surgery is an effective method of protecting healthcare workers. Most studies commend screening for COVID-19 prior to surgery, ${ }^{10}$ with most studies recommending testing at least 48 hours before the surgery. Studies also recommend quarantining the patients until test results arrive. Some

Table 2 Details of Cases Postponed Due to COVID-I9 Infection

\begin{tabular}{|l|l|l|l|l|}
\hline S.No. & Age & Diagnosis & Planned Intervention & Quarantine Period (Days) \\
\hline 1 & 62 & Open angle Glaucoma & Trabeculectomy & 14 \\
2 & 55 & Senile Cataract & Phacoemulsification & 14 \\
3 & 43 & Cortical Cataract & Phacoemulsification & 14 \\
4 & 36 & Posterior Polar Cataract & Phacoemulsification & 14 \\
5 & 57 & Senile Cataract & Phacoemulsification & 14 \\
6 & 72 & Senile Cataract & Phacoemulsification & 14 \\
7 & 61 & Posterior polar cataract & Phacoemulsification & 14 \\
8 & 75 & Mature Senile Cataract & Small incision cataract Surgery & 14 \\
9 & 42 & Pterygium & Pterygium excision & 14 \\
10 & 62 & Pterygium & Pterygium Excision & 14 \\
11 & 71 & Pterygium & Pterygium Excision & 14 \\
12 & 55 & Lid Margin Cyst & Excision & 14 \\
13 & 48 & Lid Mass & Excision & 14 \\
14 & 54 & Lid Mass & Excisional biopsy & 14 \\
15 & 58 & Lid Mass & Rectus resection & 14 \\
16 & 51 & Squint & 14 \\
\hline
\end{tabular}


recommend testing only patients with clinical signs and symptoms of COVID-19 infection or those planned for high-risk surgeries, such as organ transplant or lung resection. $^{11}$ A study by Chatterjee et al. demonstrated that doctors who examine the airways and oral cavities of patients such as otorhinolaryngologists and dentists are at a greatest risk of infection. ${ }^{12}$ Many studies emphasising screening of hospital staff for COVID-19 have demonstrated the role of asymptomatic transmission of SARSCov-2. ${ }^{13-18}$ Many studies and clinical evidence state that SARS-CoV-2 is found in the conjunctival sac of infected patients with conjunctivitis. ${ }^{19}$ Therefore eye care providers are more prone to infection by conjunctival route hence necessitating the need for eye goggles and face shield. ${ }^{20}$ Eye protection should be recommended for healthcare workers and patients in particular situations. Effective prevention guidelines are yet to be formulated to protect healthcare workers. Napoli et al. highlighted the Safety Recommendations and Medical Liability in Ocular Surgery during the COVID-19 pandemic. $^{21}$ Protective measures such as PPE kit, goggles, face shield, N95 mask and gloves are necessary. Goggles and face shield might cause technical difficulties to surgeons while operating but they provide protection against bioaerosols which are generated during the procedures.

Testing of asymptomatic patients in a containment zone is also an effective strategy. RT-PCR which is the gold standard for detecting the virus RNA, has been recommended for testing. Antibody testing is not recommended as development of antibodies takes several weeks after infection and some COVID-19 patients develop non-detectable antibodies. $^{22,23}$ Antibodies may also cross-react with other microorganisms, hence giving a false-positive result. Hence in this study we used RT-PCR for preoperative COVID screening. There is evidence that topical eye drops can inhibit viral replication hence giving false negative results. However this influence of eye drops was not taken into account in our study. $^{24}$

The latest guidelines issued by the Ministry of Health and Family Welfare (MoFHW) on August 19, 2020 state that COVID testing is not mandatory but proper history needs to be taken. If the patients is suspected/confirmed COVID positive, then elective surgery needs to be postponed. Healthcare workers need to wear appropriate personal protective equipment as per MoHFW guidelines and the operation theatre needs to be disinfected as per guidelines. $^{25}$
During our study period none of the operating doctors developed symptoms or tested positive for COVID-19. One of the nursing staff developed symptoms for novel coronavirus and later tested positive on RT-PCR testing. He was kept on home isolation with self-monitoring for 14 days.

\section{Conclusion}

This study highlights the importance of pre-operative SARS-COV-2 virus testing in preventing the spread of this virus. Our study found that 1 in 14 patients posted for surgery may be a carrier of COVID-19 infection. Healthcare workers need to be prepared as the probability of carriers showing up for elective ophthalmic surgery will be high due a high turnover of patients in ophthalmic OPD post COVID surge, even after flattening of the curve. Testing patients preoperatively is a safe strategy. Identification of COVID-19 positive patients and putting their surgery on hold until they test negative for the virus would reduce the transmission of disease to doctors, healthcare workers and other patients. Thus, universal preoperative screening is strongly recommended. ${ }^{26}$

\section{Funding}

There is no funding to report.

\section{Disclosure}

The authors report no conflicts of interest for this work.

\section{References}

1. Hussain PM, Kanwal A, Gopikrishna D. Resuming elective operations after COVID-19 pandemic. Br J Surg. 2020;107:549. doi:10.1002/ bjs. 11905

2. American College of Surgeons. Joint Statement: Roadmap for Resuming Elective Surgery after COVID-19 Pandemic. Available from: https://www.icmr.gov.in/pdf/covid/strategy/New_additional_ Advisory_23062020_3.pdf. Accessed September 8, 2021.

3. Indian Council of Medical Research.Newer Additional Strategies for COVID-19 Testing. Available from: https://www.icmr.gov.in/pdf/ COVID/strategy/New_additional_Advisory_23062020_3. pdf. Accessed September 8, 2021.

4. Sengupta S, Honavar SG, Sachdev MS, et al. All India Ophthalmological Society - Indian journal of ophthalmology consensus statement on preferred practices during the COVID-19 pandemic. Indian J Ophthalmol. 2020;68:711-724. doi:10.4103/ijo.IJO_871_20

5. American Academy of Ophthalmology. Recommendations for urgent and nonurgent patient care. San Francisco, CA, USA: American Academy of Ophthalmology; 2020.

6. British Oculoplastic Surgery Society. Update guidance on infection risk and personal protective equipment (PPE) for oculoplastic surgery during the COVID-19 pandemic. London, UK: British Oculoplastic Surgery Society; 2020. 
7. Kannan NB, Sen S, Reddy H, Kumar K, Rajan RP, Ramasamy K. Preoperative COVID-19 testing for elective vitreoretinal surgeries: experience from a major tertiary care institute in South India. Indian J Ophthalmol. 2020;68:2373-2377. doi:10.4103/ijo.IJO_2870_20

8. Sohail A, Nutini A. Forecasting the timeframe of 2019-nCoV and human cells interaction with reverse engineering. Prog Biophys Mol Biol. 2020;155:29-35. doi:10.1016/j.pbiomolbio. 2020.04.002

9. Kaye K, Paprottka F, Escudero R, et al. Elective, non-urgent procedures and aesthetic surgery in the wake of SARS-COVID-19: considerations regarding safety, feasibility and impact on clinical management. Aesthetic Plast Surg. 2020;44:1014-1042. doi:10. 1007/s00266-020-01752-9

10. Honavar SG. Nosocomial COVID-19 transmission in routine ophthalmic practice-Is there new evidence? Indian J Ophthalmol. 2020;68:2059-2060. doi:10.4103/ijo.IJO_3046_20

11. Morris M, Pierce A, Carlisle B, Vining B, Dobyns J. Pre-operative COVID-19 testing and decolonization. Am J Surg. 2020;220 558-560. doi:10.1016/j.amjsurg.2020.05.027

12. Chatterjee P, Anand T, Singh KJ, et al. Healthcare workers \& SARSCoV-2 infection in India: a case-control investigation in the time of COVID-19. Indian J Med Res. 2020;151:459-467. doi:10.4103/ijmr. IJMR_2234_20

13. Korth J, Wilde B, Dolff S, et al. SARS-CoV-2-specific antibody detection in healthcare workers in Germany with direct contact to COVID-19 patients. J Clin Virol. 2020;128:104437. doi:10.1016/j. jcv.2020.104437

14. Bendavid E, Mulaney B, Sood N, et al. COVID-19 antibody seroprevalence in Santa Clara County, California. Int $J$ Epidemiol. 2021;50:410-419. doi:10.1093/ije/dyab010

15. Steensels D, Oris E, Coninx L, et al. Hospital-wide SARS-CoV-2 antibody screening in 3056 staff in a tertiary center in Belgium. JAMA. 2020;324:195-197. doi:10.1001/jama.2020. 11160

16. Poulikakos D, Sinha S, Kalra PA. SARS-CoV-2 antibody screening in healthcare workers in a tertiary centre in North West England. J Clin Virol. 2020;129:104545. doi:10.1016/j.jcv.2020. 104545
17. Kluytmans-van den Bergh MFQ, Buiting AGM, Pas SD, et al. Prevalence and clinical presentation of health care workers with symptoms of coronavirus disease 2019 in 2 Dutch hospitals during an early phase of the pandemic. JAMA Netw Open. 2020;3:209673. doi:10.1001/jamanetworkopen.2020.9673

18. Keeley AJ, Evans C, Colton H, et al. Roll-out of SARS-CoV-2 testing for healthcare workers at a large NHS foundation trust in the United Kingdom, March 2020. Euro Surveill. 2020;25:2000433. doi:10.2807/1560-7917.ES.2020.25.14.2000433

19. Xia J, Tong J, Liu M, Shen Y, Guo D. Evaluation of coronavirus in tears and conjunctival secretions of patients with SARS-CoV-2 infection. J Med Virol. 2020;92:589-594. doi:10.1002/jmv.25725

20. Napoli PE, Nioi M, d'Aloja E, Fossarello M. The ocular surface and the coronavirus disease 2019: does a dual 'ocular route' exist? J Clin Med. 2020;9:1269. doi:10.3390/jcm9051269

21. Napoli PE, Nioi M, d'Aloja E, Fossarello M. Safety recommendations and medical liability in ocular surgery during the COVID-19 pandemic: an unsolved dilemma. J Clin Med. 2020;9:1403. doi:10.3390/jcm9051403

22. Zhao J, Yuan Q, Wang H, et al. Antibody responses to SARS-CoV-2 in patients of novel coronavirus disease 2019. Clin Infect Dis. 2020;71:2027-2034. doi:10.1093/cid/ciaa344

23. Okba NMA, Müller MA, Li W, et al. Severe acute respiratory syndrome coronavirus 2-specific antibody responses in coronavirus disease patients. Emerg Infect Dis. 2020;26:1478-1488. doi:10.3201/eid2607.200841

24. Napoli PE, Mangoni L, Gentile P, Braghiroli M, Fossarello M. A panel of broad-spectrum antivirals in topical ophthalmic medications from the drug repurposing approach during and after the coronavirus disease 2019 era. J Clin Med. 2020;9:2441. doi:10.3390/jcm9082441

25. Ministry of Health and Family Welfare, Government of India. Guidelines on Safe Ophthalmology Practices in Covid-19 Scenario. Available from: https://www.mohfw.gov.in/pdf/ GuidelinesonSafeOphthalmologyPracticesinCovid19Scenario.pdf. Accessed September 8, 2021.

26. Goel R, Arora R, Khanam S, Saxena S, Manchanda V, Pumma P. Is it essential to perform COVID-19 testing prior to ophthalmic procedures? Indian J Ophthalmol. 2020;68:2335-2337. doi:10.4103/ijo.IJO_3020_20
Clinical Ophthalmology

\section{Publish your work in this journal}

Clinical Ophthalmology is an international, peer-reviewed journal covering all subspecialties within ophthalmology. Key topics include: Optometry; Visual science; Pharmacology and drug therapy in eye diseases; Basic Sciences; Primary and Secondary eye care; Patient Safety and Quality of Care Improvements. This journal is indexed on PubMed
Dovepress

Central and CAS, and is the official journal of The Society of Clinical Ophthalmology (SCO). The manuscript management system is completely online and includes a very quick and fair peer-review system, which is all easy to use. Visit http://www.dovepress.com/ testimonials.php to read real quotes from published authors. 